\title{
On zero sum-partition of Abelian groups into three sets and group distance magic labeling
}

\author{
Sylwia Cichacz \\ Faculty of Applied Mathematics, AGH University of Science and Technology, \\ Al. Mickiewicza 30, 30-059 Kraków, Poland
}

Received 2 March 2016, accepted 1 May 2017, published online 10 May 2017

\begin{abstract}
We say that a finite Abelian group $\Gamma$ has the constant-sum-partition property into $t$ sets $\left(\operatorname{CSP}(t)\right.$-property) if for every partition $n=r_{1}+r_{2}+\ldots+r_{t}$ of $n$, with $r_{i} \geq 2$ for $2 \leq i \leq t$, there is a partition of $\Gamma$ into pairwise disjoint subsets $A_{1}, A_{2}, \ldots, A_{t}$, such that $\left|A_{i}\right|=r_{i}$ and for some $\nu \in \Gamma, \sum_{a \in A_{i}} a=\nu$ for $1 \leq i \leq t$. For $\nu=g_{0}$ (where $g_{0}$ is the identity element of $\Gamma$ ) we say that $\Gamma$ has zero-sum-partition property into $t$ sets (ZSP $(t)$-property).

A $\Gamma$-distance magic labeling of a graph $G=(V, E)$ with $|V|=n$ is a bijection $\ell$ from $V$ to an Abelian group $\Gamma$ of order $n$ such that the weight $w(x)=\sum_{y \in N(x)} \ell(y)$ of every vertex $x \in V$ is equal to the same element $\mu \in \Gamma$, called the magic constant. A graph $G$ is called a group distance magic graph if there exists a $\Gamma$-distance magic labeling for every Abelian group $\Gamma$ of order $|V(G)|$.

In this paper we study the $\operatorname{CSP}(3)$-property of $\Gamma$, and apply the results to the study of group distance magic complete tripartite graphs.
\end{abstract}

Keywords: Abelian group, constant sum partition, group distance magic labeling.

Math. Subj. Class.: 05C25, $05 C 78$

\section{Introduction}

All graphs considered in this paper are simple finite graphs. Consider a simple graph $G$ whose order we denote by $n=|G|$. We denote by $V(G)$ the vertex set and $E(G)$ the edge set of a graph $G$. The open neighborhood $N(x)$ of a vertex $x$ is the set of vertices adjacent to $x$, and the degree $d(x)$ of $x$ is $|N(x)|$, the size of the neighborhood of $x$.

E-mail address: cichacz@agh.edu.pl (Sylwia Cichacz) 
Let the identity element of $\Gamma$ be denoted by $g_{0}$. Recall that any group element $\iota \in \Gamma$ of order 2 (i.e., $\iota \neq g_{0}$ such that $2 \iota=g_{0}$ ) is called an involution.

In [8] Kaplan, Lev and Roditty introduced a notion of zero-sum partitions of subsets in Abelian groups. Let $\Gamma$ be an Abelian group and let $A$ be a finite subset of $\Gamma-\left\{g_{0}\right\}$, with $|A|=n-1$. We shall say that $A$ has the zero-sum-partition property (ZSP-property) if every partition $n-1=r_{1}+r_{2}+\ldots+r_{t}$ of $n-1$, with $r_{i} \geq 2$ for $1 \leq i \leq t$ and for any possible positive integer $t$, there is a partition of $A$ into pairwise disjoint subsets $A_{1}, A_{2}, \ldots, A_{t}$, such that $\left|A_{i}\right|=r_{i}$ and $\sum_{a \in A_{i}} a=g_{0}$ for $1 \leq i \leq t$. In the case that $\Gamma$ is finite, we shall say that $\Gamma$ has the ZSP-property if $A=\Gamma-\left\{g_{0}\right\}$ has the ZSP-property.

They proved the following theorem for cyclic groups of odd order.

Theorem 1.1 ([8]). The group $\mathbb{Z}_{n}$ has the ZSP-property if and only if $n$ is odd.

Moreover, Kaplan, Lev and Roditty showed that if $\Gamma$ is a finite Abelian group of even order $n$ such that the number of involutions in $\Gamma$ is different from 3 , then $\Gamma$ does not have the ZSP-property [8]. Their results along with results proved by Zeng [10] give necessary and sufficient conditions for the ZSP-property for a finite Abelian group.

Theorem 1.2 ([8, 10]). Let $\Gamma$ be a finite Abelian group. Then $\Gamma$ has the ZSP-property if and only if either $\Gamma$ is of odd order or $\Gamma$ contains exactly three involutions.

They apply those results to the study of anti-magic trees $[8,10]$.

We generalize the notion of ZSP-property. We say that a finite Abelian group $\Gamma$ has the constant-sum-partition property into t sets $(\operatorname{CSP}(t)$-property) if for every partition $n=$ $r_{1}+r_{2}+\ldots+r_{t}$ of $n$, with $r_{i} \geq 2$ for $2 \leq i \leq t$, there is a partition of $\Gamma$ into pairwise disjoint subsets $A_{1}, A_{2}, \ldots, A_{t}$, such that $\left|A_{i}\right|=r_{i}$ and for some $\nu \in \Gamma, \sum_{a \in A_{i}} a=\nu$ for $1 \leq i \leq t$. For $\nu=g_{0}$ we say that $\Gamma$ has zero-sum-partition property into $t$ sets $(Z S P(t)$-property).

In this paper we investigate also distance magic labelings, which belong to a large family of magic type labelings.

A distance magic labeling (also called sigma labeling) of a graph $G=(V, E)$ of order $n$ is a bijection $\ell: V \rightarrow\{1,2, \ldots, n\}$ with the property that there is a positive integer $k$ (called the magic constant) such that

$$
w(x)=\sum_{y \in N(x)} \ell(y)=k \text { for every } x \in V(G),
$$

where $w(x)$ is the weight of vertex $x$. If a graph $G$ admits a distance magic labeling, then we say that $G$ is a distance magic graph.

The concept of distance magic labeling has been motivated by the construction of magic rectangles, since we can construct a distance magic complete $r$-partite graph with each part size equal to $n$ by labeling the vertices of each part by the columns of the magic rectangle. Although there does not exist a $2 \times 2$ magic rectangle, observe that the partite sets of $K_{2,2}$ can be labeled $\{1,4\}$ and $\{2,3\}$, respectively, to obtain a distance magic labeling. The following result was proved in [9].

Observation 1.3 ([9]). There is no distance magic $r$-regular graph with $r$ odd. 
Froncek in [7] defined the notion of group distance magic graphs, i.e., the graphs allowing a bijective labeling of vertices with elements of an Abelian group resulting in constant sums of neighbor labels.

A $\Gamma$-distance magic labeling of a graph $G=(V, E)$ with $|V|=n$ is a bijection $\ell$ from $V$ to an Abelian group $\Gamma$ of order $n$ such that the weight $w(x)=\sum_{y \in N(x)} \ell(y)$ of every vertex $x \in V$ is equal to the same element $\mu \in \Gamma$, called the magic constant. A graph $G$ is called a group distance magic graph if there exists a $\Gamma$-distance magic labeling for every Abelian group $\Gamma$ of order $|V(G)|$.

The connection between distance magic graphs and $\Gamma$-distance magic graphs is as follows. Let $G$ be a distance magic graph of order $n$ with the magic constant $\mu^{\prime}$. If we replace the label $n$ in a distance magic labeling for the graph $G$ by the label 0 , then we obtain a $\mathbb{Z}_{n^{-}}$ distance magic labeling for the graph $G$ with the magic constant $\mu=\mu^{\prime}(\bmod n)$. Hence every distance magic graph with $n$ vertices admits a $\mathbb{Z}_{n}$-distance magic labeling. However a $\mathbb{Z}_{n}$-distance magic graph on $n$ vertices is not necessarily a distance magic graph. Moreover, there are some graphs that are not distance magic while at the same time they are group distance magic (see [4]).

A general theorem for $\Gamma$-distance magic labeling similar to Observation 1.3 was proved recently.

Theorem 1.4 ([5]). Let $G$ be an r-regular graph on $n$ vertices, where $r$ is odd. There does not exist an Abelian group $\Gamma$ of order $n$ with exactly one involution $\iota$ such that $G$ is $\Gamma$-distance magic.

Notice that the constant sum partitions of a group $\Gamma$ lead to complete multipartite $\Gamma$-distance magic labeled graphs. For instance, the partition $\{0\},\{1,2,4\},\{3,5,6\}$ of the group $\mathbb{Z}_{7}$ with constant sum 0 leads to a $\mathbb{Z}_{7}$-distance magic labeling of the complete tripartite graph $K_{1,3,3}$. More general, let $G$ be a complete $t$-partite graph of order $n$ with the partition sets $V_{1}, V_{2}, \ldots, V_{t}$. Note that $G$ is $\Gamma$-distance magic if and only if $\sum_{i=1, i \neq j}^{t} \sum_{x \in V_{i}} \ell(x)=\mu$ for $j \in\{1,2, \ldots, t\}$ which implies that $\sum_{x \in V_{j}} \ell(x)=\nu$ for $j \in\{1,2, \ldots, t\}$ and some $\nu \in \Gamma$. Therefore we can see that $G$ is $\Gamma$-distance magic if and only if $\Gamma$ has the $\operatorname{CSP}(t)$-property. The following theorems were proven in [3].

Theorem 1.5 ([3]). Let $G=K_{n_{1}, n_{2}, \ldots, n_{t}}$ be a complete $t$-partite graph and $n=n_{1}+$ $n_{2}+\ldots+n_{t}$. If $n \equiv 2(\bmod 4)$ and $t$ is even, then there does not exist an Abelian group $\Gamma$ of order $n$ such that $G$ is a $\Gamma$-distance magic graph.

Theorem 1.6 ([3]). The complete bipartite graph $K_{n_{1}, n_{2}}$ is a group distance magic graph if and only if $n_{1}+n_{2} \not \equiv 2(\bmod 4)$.

Therefore it follows that an Abelian group $\Gamma$ of order $n$ has the $\operatorname{CSP}(2)$-property if and only if $n \not \equiv 2(\bmod 4)$.

In this paper we study the $\operatorname{CSP}(3)$-property of $\Gamma$, and apply the results to an investigation of the necessary and sufficient conditions for complete tripartite graphs to be group distance magic. This work will also be potentially useful for group theorists working on Abelian groups. 


\section{Preliminaries}

Assume $\Gamma$ is an Abelian group of order $n$ with the operation denoted by + . For convenience we will write $k a$ to denote $a+a+\ldots+a$ (where the element $a$ appears $k$ times), $-a$ to denote the inverse of $a$ and we will use $a-b$ instead of $a+(-b)$. Recall that a non-trivial finite group has elements of order 2 if and only if the order of the group is even. The fundamental theorem of finite Abelian groups states that a finite Abelian group $\Gamma$ of order $n$ can be expressed as the direct product of cyclic subgroups of prime-power order. This implies that

$$
\Gamma \cong \mathbb{Z}_{p_{1}^{\alpha_{1}}} \times \mathbb{Z}_{p_{2}^{\alpha_{2}}} \times \ldots \times \mathbb{Z}_{p_{k}^{\alpha_{k}}} \quad \text { where } n=p_{1}^{\alpha_{1}} \cdot p_{2}^{\alpha_{2}} \cdot \ldots \cdot p_{k}^{\alpha_{k}}
$$

and $p_{i}$ for $i \in\{1,2, \ldots, k\}$ are not necessarily distinct primes. This product is unique up to the order of the direct product. When $t$ is the number of these cyclic components whose order is a multiple of 2 , then $\Gamma$ has $2^{t}-1$ involutions. In particular, if $n \equiv 2(\bmod 4)$, then $\Gamma \cong \mathbb{Z}_{2} \times A$ for some Abelian group $A$ of odd order $n / 2$. Moreover every cyclic group of even order has exactly one involution. The sum of all the group elements is equal to the sum of the involutions and the neutral element.

The following lemma was proved in [6] (see [6], Lemma 8).

Lemma 2.1 ([6]). Let $\Gamma$ be an Abelian group.

1. If $\Gamma$ has exactly one involution $\iota$, then $\sum_{g \in \Gamma} g=\iota$.

2. If $\Gamma$ has no involutions, or more than one involution, then $\sum_{g \in \Gamma} g=g_{0}$.

Anholcer and Cichacz proved the following (see [1], Lemma 2.4).

Lemma 2.2 ([1]). Let $\Gamma$ be an Abelian group with involutions set $I^{*}=\left\{\iota_{1}, \iota_{2}, \ldots, \iota_{2^{k}-1}\right\}, k>1$ and let $I=I^{*} \cup\left\{g_{0}\right\}$. Given positive integers $n_{1}$, $n_{2}$ such that $n_{1}+n_{2}=2^{k}$. There exists a partition $A=\left\{A_{1}, A_{2}\right\}$ of I such that

1. $n_{1}=\left|A_{1}\right|, n_{2}=\left|A_{2}\right|$,

2. $\sum_{a \in A_{i}} a=g_{0}$ for $i=1,2$,

if and only if none of $n_{1}, n_{2}$ is 2 .

\section{Constant sum partition of Abelian groups}

Note that if $\Gamma$ has odd order, then it has the ZSP-property by Theorem 1.2, thus one can check that it has the ZSP(3)-property. We now generalize Lemma 2.2.

Lemma 3.1. Let $\Gamma$ be an Abelian group with involutions set $I^{*}=\left\{\iota_{1}, \iota_{2}, \ldots, \iota_{2^{k}-1}\right\}$, $k>2$ and let $I=I^{*} \cup\left\{g_{0}\right\}$. Given positive integers $n_{1}, n_{2}, n_{3}$ such that $n_{1}+n_{2}+n_{3}=2^{k}$. There exists a partition $A=\left\{A_{1}, A_{2}, A_{3}\right\}$ of I such that

1. $n_{1}=\left|A_{1}\right|, n_{2}=\left|A_{2}\right|, n_{3}=\left|A_{3}\right|$,

2. $\sum_{a \in A_{i}} a=g_{0}$ for $i \in\{1,2,3\}$,

if and only if $n_{1}, n_{2}, n_{3} \notin\left\{2,2^{k}-2\right\}$. 
Proof. For $n_{i}=n_{j}=1$ we have that $\sum_{a \in A_{i}} a \neq \sum_{a \in A_{j}} a$. For $n_{i}=2$, it is easy to see $\sum_{a \in A_{i}} a \neq g_{0}$.

Let $\iota_{0}=g_{0}$. Recall that since $I=\left\{\iota_{0}, \iota_{1}, \ldots, \iota_{2^{k}-1}\right\}$ is a subgroup of $\Gamma$, we have $I \cong\left(\mathbb{Z}_{2}\right)^{k}$. One can check that the lemma is true for $k \in\{3,4\}$. The sufficiency will be proved then by induction on $k$. Namely, suppose the assertion is true for some $m=$ $k \geq 4$. We want to prove it is true for $m=k+1$. Let $\left(n_{1}, n_{2}, n_{3}\right)$ be a triple such that $n_{1}, n_{2}, n_{3} \notin\left\{2,2^{k+1}-2\right\}$ and $n_{1}+n_{2}+n_{3}=2^{k+1}$. For $i \in\{1,2,3\}$ let $n_{i}=4 q_{i}+r_{i}$, where $r_{i} \in\{1,3,4,5,6\}$ and 1 appears at most once as a value of some $r_{i}$. Observe that $r_{1}+r_{2}+r_{3} \leq 18$, but because $n_{1}+n_{2}+n_{3} \equiv 0(\bmod 4)$ and $n_{1}+n_{2}+n_{3}=$ $4\left(q_{1}+q_{2}+q_{3}\right)+r_{1}+r_{2}+r_{3}$, we must have $r_{1}+r_{2}+r_{3} \equiv 0(\bmod 4)$, which implies that $r_{1}+r_{2}+r_{3} \leq 16$. Thus $4\left(q_{1}+q_{2}+q_{3}\right) \geq 2^{k}$.

Now we select $t_{1}, t_{2}, t_{3}$ such that $t_{i} \leq q_{i}$ and $4\left(t_{1}+t_{2}+t_{3}\right)=2^{k}$. Denote $n_{i}^{\prime}=n_{i}-4 t_{i}$ and $n_{i}^{\prime \prime}=4 t_{i}$. Obviously, $n_{1}^{\prime}+n_{2}^{\prime}+n_{3}^{\prime}=n_{1}^{\prime \prime}+n_{2}^{\prime \prime}+n_{3}^{\prime \prime}=2^{k}$ and $n_{i}^{\prime} \notin\left\{0,2,2^{k}-2\right\}$ for any $i \in\{1,2,3\}$.

If also $n_{i}^{\prime \prime} \neq 0$, then both triples $\left(n_{1}^{\prime}, n_{2}^{\prime}, n_{3}^{\prime}\right)$ and $\left(n_{1}^{\prime \prime}, n_{2}^{\prime \prime}, n_{3}^{\prime \prime}\right)$ satisfy the inductive hypothesis and there exist partitions of $\left(\mathbb{Z}_{2}\right)^{k}$ into sets $S_{1}^{\prime}, S_{2}^{\prime}, S_{3}^{\prime}$ and $S_{1}^{\prime \prime}, S_{2}^{\prime \prime}, S_{3}^{\prime \prime}$ of respective orders $n_{1}^{\prime}, n_{2}^{\prime}, n_{3}^{\prime}$ and $n_{1}^{\prime \prime}, n_{2}^{\prime \prime}, n_{3}^{\prime \prime}$. If we now replace each element $\left(x_{1}, x_{2}, \ldots, x_{k}\right)$ of $\left(\mathbb{Z}_{2}\right)^{k}$ in any $S_{i}^{\prime}$ by the $\left(x_{1}, x_{2}, \ldots, x_{k}, 0\right)$ of $\left(\mathbb{Z}_{2}\right)^{k+1}$, it should be clear that the sum of elements in each $S_{i}^{\prime}$ is the identity of $\left(\mathbb{Z}_{2}\right)^{k+1}$.

Similarly, we replace each element $\left(y_{1}, y_{2}, \ldots, y_{k}\right)$ of $\left(\mathbb{Z}_{2}\right)^{k}$ in any $S_{i}^{\prime \prime}$ by the element $\left(y_{1}, y_{2}, \ldots, y_{k}, 1\right)$ of $\left(\mathbb{Z}_{2}\right)^{k+1}$. Now because the order of each $S_{i}^{\prime \prime}$ is even, the ones in last entries add up to zero and the sum of elements in each $S_{i}^{\prime \prime}$ is again the identity of $\left(\mathbb{Z}_{2}\right)^{k+1}$. Now set $S_{i}=S_{i}^{\prime} \cup S_{i}^{\prime \prime}$ to obtain the desired partition of $\left(\mathbb{Z}_{2}\right)^{k+1}$.

The case when $n_{i}^{\prime \prime}=0$ and $n_{j}^{\prime \prime}, n_{l}^{\prime \prime} \neq 0$ can be treated using Lemma 2.2, and the case when $n_{i}^{\prime \prime}=n_{j}^{\prime \prime}=0$ and $n_{l}^{\prime \prime}=2^{k}$ is obvious.

Theorem 3.2. Let $\Gamma$ be an Abelian group of even order $n$. $\Gamma$ has the $\operatorname{CSP}(3)$-property if and only if $\Gamma \neq\left(\mathbb{Z}_{2}\right)^{t}$ for some positive integer $t$. Moreover, $\Gamma \neq\left(\mathbb{Z}_{2}\right)^{t}$ has the $\operatorname{ZSP}(3)$ property if and only if $\Gamma$ has more than one involution.

Proof. For a given partition $n=n_{1}+n_{2}+n_{3}$ we will construct a partition $\Gamma=A_{1} \cup A_{2} \cup A_{3}$ such that $A_{i}=\left\{a_{0}^{i}, a_{1}^{i}, \ldots, a_{n_{i}-1}^{i}\right\}$ for $i \in\{1,2,3\}$. Let $\Gamma=\left\{g_{0}, g_{1}, \ldots, g_{n-1}\right\}$. Recall that by $g_{0}$ we denote the identity element of $\Gamma$.

Assume first that $\Gamma \cong\left(\mathbb{Z}_{2}\right)^{t}$ for $t>1$ has the $\operatorname{CSP}(3)$-property. Let $A_{1}, A_{2}, A_{3}$ be the desired partition of $\Gamma$ for $n_{2}=2$. Hence $\sum_{a \in A_{i}} a=\iota \neq g_{0}$ for $i \in\{1,2,3\}$. Therefore $\sum_{g \in \Gamma} g=\sum_{i=1}^{3} \sum_{a \in A_{i}} a=3 \iota=\iota \neq g_{0}$, a contradiction with Lemma 2.1.

Suppose now that $\Gamma$ has the $\operatorname{ZSP}(3)$-property and there is the only one involution $\iota \in \Gamma$. Let $A_{1}, A_{2}, A_{3}$ be the desired partition of $\Gamma$, therefore $\sum_{a \in A_{i}} a=g_{0}$ for $i \in\{1,2,3\}$. Hence, $g_{0}=\sum_{i=1}^{3} \sum_{a \in A_{i}} a=\sum_{g \in \Gamma} g$, on the other hand by Lemma 2.1 we have $\sum_{g \in \Gamma} g=\iota$, a contradiction.

We will prove sufficiency now. Let us consider two cases on the number of involutions in $\Gamma$. 
Case 1. There is exactly one involution $\iota$ in $\Gamma$.

Notice that in that case $|\Gamma| \geq 6$. By fundamental theorem of finite Abelian groups

$\Gamma \cong \mathbb{Z}_{2^{\alpha_{1}}} \times \mathbb{Z}_{p_{2}^{\alpha_{2}}} \times \ldots \times \mathbb{Z}_{p_{k}^{\alpha_{k}}} \quad$ where $n=2^{\alpha_{1}} \cdot p_{2}^{\alpha_{2}} \cdot \ldots \cdot p_{k}^{\alpha_{k}}, \quad \alpha_{1} \geq 1$

and $p_{i} \geq 3$ for $i \in\{2,3, \ldots, k\}$ are not necessarily distinct primes. Since $|\Gamma| \geq 6$ we have $\Gamma \cong \mathbb{Z}_{2 m} \times A$ for $m \geq 3$ and some Abelian group $A$ of order $n / 2 m$. Let $g_{1}=\iota$ and $g_{i+1}=-g_{i}$ for $i \in\{2,4,6, \ldots, n-2\}$. Using the isomorphism $\varphi: \Gamma \rightarrow \mathbb{Z}_{2 m} \times A$, we can identify every $g \in \Gamma$ with its image $\varphi(g)=\left(j, a_{i}\right)$, where $j \in \mathbb{Z}_{2 m}$ and $a_{i} \in A$ for $i \in\left\{0,1, \ldots, \frac{n}{2 m}-1\right\}$ and $a_{0}$ is the identity element in $A$. Observe that $g_{1}=\iota=\left(m, a_{0}\right)$. Because $m>2$ we can set $g_{2}=\left(1, a_{0}\right), g_{3}=\left(2 m-1, a_{0}\right), g_{4}=\left(m-1, a_{0}\right)$, $g_{5}=\left(m+1, a_{0}\right)$.

Without loss of generality we can assume that $n_{1}$ is even and $n_{2} \geq n_{3}$. Let $a_{0}^{1}=g_{2}$, $a_{1}^{1}=g_{4}$ and $a_{i}^{1}=g_{i+4}$ for $i \in\left\{2,3, \ldots, n_{1}-1\right\}$.

Case 1.1. $n_{2}, n_{3}$ are both odd.

Let: $a_{0}^{2}=g_{0}, a_{1}^{2}=g_{3}, a_{2}^{2}=g_{5}$ and $g_{i}^{1}=a_{n_{1}+1+i}$ for $i \in\left\{3,4, \ldots, n_{2}-1\right\}$.

$a_{0}^{3}=g_{1}$ and $a_{i}^{3}=g_{n_{1}+n_{2}+i}$ for $i \in\left\{1,2, \ldots, n_{3}-1\right\}$.

Case 1.2. $n_{2}, n_{3}$ are both even.

Let: $a_{0}^{2}=g_{3}, a_{1}^{2}=g_{5}$ and $a_{i}^{1}=g_{n_{1}+2+i}$ for $i \in\left\{2,3, \ldots, n_{2}-1\right\}$.

$a_{0}^{3}=g_{0}, a_{1}^{3}=g_{1}$ and $a_{i}^{3}=g_{n_{1}+n_{2}+i}$ for $i \in\left\{2,3, \ldots, n_{3}-1\right\}$.

Note that in both Cases 1.1 and 1.2 we obtain that $\sum_{a \in A^{i}} a=\left(m, a_{0}\right)=\iota$ for $i \in\{1,2,3\}$.

Case 2. There is more that one involution $\iota$ in $\Gamma$.

By fundamental theorem of finite Abelian groups $\Gamma$ has $2^{t}-1$ involutions $\iota_{1}, \iota_{2}, \ldots, \iota_{2} 2_{-1}$ for $t>1$. Let $g_{i}=\iota_{i}$ for $i \in\left\{1,2, \ldots, 2^{t}-1\right\}$, and $g_{i+1}=-g_{i}$ for $i \in\left\{2^{t}, 2^{t}+2,2^{t}+\right.$ $4, \ldots, n-2\}$. By the above arguments on necessity we obtain that $\Gamma \neq\left(\mathbb{Z}_{2}\right)^{t}$, therefore $2^{t} \leq n / 2$. One can check, that we can choose integers $t_{1}, t_{2}$ and $t_{3}$ such that:

$$
t_{1}+t_{2}+t_{3}=2^{t}
$$

with

$$
n_{i}-t_{i} \equiv 0 \quad(\bmod 2), \quad t_{i} \geq 0, \quad t_{i} \notin\left\{2,2^{t}-2\right\} \quad \text { for } \quad i \in\{1,2,3\} .
$$

By Lemmas 2.2 and 3.1 it follows that there exists a partition $B=\left\{B_{1}, B_{2}, B_{3}\right\}$ of $I=\left\{g_{0}, g_{1}, \ldots, g_{2^{t}-1}\right\}$ such that $t_{1}=\left|B_{1}\right|, t_{2}=\left|B_{2}\right|, t_{3}=\left|B_{3}\right|$, and if $B_{i} \neq \emptyset$, then $\sum_{b \in B_{i}} b=g_{0}$ for $i \in\{1,2,3\}$. Let $B_{i}=\left\{b_{0}^{i}, b_{1}^{i}, \ldots, b_{t_{i}-1}^{i}\right\}$ for $i \in\{1,2,3\}$. Let us set now:

$a_{i}^{1}=b_{i}^{1}$ for $i \in\left\{1,2, \ldots, t_{1}-1\right\}$ and $a_{i}^{1}=g_{i+t_{2}+t_{3}}$ for $i \in\left\{t_{1}, t_{1}+1 \ldots, n_{1}-1\right\}$, $a_{i}^{2}=b_{i}^{2}$ for $i \in\left\{1,2, \ldots, t_{2}-1\right\}$ and $a_{i}^{2}=g_{i+t_{3}+n_{1}}$ for $i \in\left\{t_{2}, t_{2}+1 \ldots, n_{2}-1\right\}$, $a_{i}^{3}=b_{i}^{3}$ for $i \in\left\{1,2, \ldots, t_{3}-1\right\}$ and $a_{i}^{3}=g_{i+n_{1}+n_{2}}$ for $i \in\left\{t_{3}, t_{3}+1 \ldots, n_{3}-1\right\}$. In this case $\sum_{a \in A_{i}} a=g_{0}$ for $i \in\{1,2,3\}$.

\section{Group distance magic graphs}

Observe that for $G$ being an odd regular graph of order $n$, by hand shaking lemma $n$ is even. Thus, the below theorem is a generalization of Theorem 1.4. 
Theorem 4.1. Let $G$ have order $n \equiv 2(\bmod 4)$ with all vertices having odd degree. There does not exist an Abelian group $\Gamma$ of order $n$ such that $G$ is a $\Gamma$-distance magic graph.

Proof. Assumption $n \equiv 2(\bmod 4)$ implies that $\Gamma \cong \mathbb{Z}_{2} \times A$ for some Abelian group $A$ of odd order $n / 2$ and there exists exactly one involution $\iota \in \Gamma$. Let $g_{n / 2}=\iota, g_{n / 2+i}=-a_{i}$ for $i \in\{1,2, \ldots, n / 2-1\}$. Let $V(G)=\left\{x_{0}, x_{1}, \ldots, x_{n-1}\right\}$.

Suppose that $\ell$ is a $\Gamma$-distance labeling for $G$ and $\mu$ is the magic constant. Without loss of generality we can assume that $\ell\left(x_{i}\right)=a_{i}$ for $i \in\{0,1, \ldots, n-1\}$. Recall that $n g=0$ for any $g \in \Gamma$ and $\operatorname{deg}\left(x_{n / 2}\right) g_{n / 2}=g_{n / 2}=\iota$ since $\operatorname{deg}\left(x_{n / 2}\right)$ is odd. Notice that $\operatorname{deg}\left(x_{i}\right)-\operatorname{deg}\left(x_{n-i}\right)=2 d_{i}$ for some integer $d_{i}$ for $i \in\{1,2, \ldots, n / 2-1\}$, because all vertices have odd degree. Let now

$$
\begin{gathered}
w(G)=\sum_{x \in V(G)} \sum_{y \in N(x)} w(y)=\sum_{i=0}^{n-1} \operatorname{deg}\left(x_{i}\right) g_{i}= \\
\sum_{i=1}^{n / 2-1} \operatorname{deg}\left(x_{i}\right) g_{i}+\operatorname{deg}\left(x_{n / 2}\right) g_{n / 2}+\sum_{i=1}^{n / 2-1} \operatorname{deg}\left(x_{n-i}\right) g_{n-i}= \\
\sum_{i=1}^{n / 2-1} \operatorname{deg}\left(x_{i}\right) g_{i}-\sum_{i=1}^{n / 2-1} \operatorname{deg}\left(x_{n-i}\right) g_{i}+g_{n / 2}= \\
\sum_{i=1}^{n / 2-1}\left(\operatorname{deg}\left(x_{i}\right)-\operatorname{deg}\left(x_{n-i}\right)\right) g_{i}+g_{n / 2}=2 \sum_{i=1}^{n / 2-1} d_{i} g_{i}+g_{n / 2}
\end{gathered}
$$

On the other hand, $w(G)=\sum_{x \in V(G)} w(x)=n \cdot \mu=g_{0}$. Therefore we obtain that $2 v=g_{n / 2}$ for some element $v \in \Gamma$. Since $n / 2$ is odd and $\Gamma \cong \mathbb{Z}_{2} \times A$, such an element $v$ does not exist, a contradiction.

From the above Theorem 4.1 we obtain the following.

Theorem 4.2. If $G$ have order $n \equiv 2(\bmod 4)$ with all vertices having odd degree, then $G$ is not distance magic.

Proof. The graph $G$ is not $\mathbb{Z}_{n}$-distance magic by Theorem 4.1 , therefore it is not distance magic.

We prove now the following useful lemma.

Lemma 4.3. Let $G=K_{n_{1}, n_{2}, \ldots, n_{t}}$ be a complete $t$-partite graph and $n=n_{1}+n_{2}+\ldots+$ $n_{t}$. If $n_{1} \leq n_{2} \leq \ldots \leq n_{t}$ and $n_{2}=1$, then there does not exist an Abelian group $\Gamma$ of order $n$ such that $G$ is a $\Gamma$-distance magic graph.

Proof. Let $G$ have the partition vertex sets $V_{i}$ such that $\left|V_{i}\right|=n_{i}$ for $i \in\{1,2, \ldots, t\}$. Let $x \in V_{1}$ and $y \in V_{2}$. Suppose that the graph $G$ is $\Gamma$-distance magic for some Abelian group $\Gamma$ of order $n$ and that $\ell$ is a $\Gamma$-distance magic labeling of $G$, then $w(x)=\sum_{g \in \Gamma} g-\ell(x)=$ $w(y)=\sum_{g \in \Gamma} g-\ell(y)$. Thus $\ell(y)=\ell(x)$, a contradiction. 
Theorem 4.4. Let $G=K_{n_{1}, n_{2}, n_{3}}$ be a complete tripartite graph such that $1 \leq n_{1} \leq n_{2} \leq$ $n_{3}$ and $n=n_{1}+n_{2}+n_{3}$. The graph $G$ is a group distance magic graph if and only if $n_{2}>1$ and $n_{1}+n_{2}+n_{3} \neq 2^{p}$ for any positive integer $p$.

Proof. Let $G$ have the partition vertex sets $V_{i}$ such that $\left|V_{i}\right|=n_{i}$ for $i \in\{1,2,3\}$. We can assume that $n_{2}>1$ by Lemma 4.3.

Suppose now that $\Gamma \cong\left(\mathbb{Z}_{2}\right)^{p}$ for some integer $p$. Let $n_{1}=2$ and $\ell$ be a $\Gamma$-distance magic labeling of $G$. Thus $\sum_{x \in V_{1}} \ell(x)=\iota \neq g_{0}$. Since $G$ is $\Gamma$-distance magic we obtain that $\sum_{x \in V_{i}} \ell(x)=\iota$ for $i \in\{1,2,3\}$. Therefore $\sum_{g \in \Gamma} g=\sum_{i=1}^{3} \sum_{x \in V_{i}} \ell(x)=3 \iota=$ $\iota \neq g_{0}$, a contradiction with Lemma 2.1 .

If $\Gamma \neq\left(\mathbb{Z}_{2}\right)^{p}$ and $n_{i} \geq 2$ for $i \in\{2,3\}$, then the group $\Gamma$ can be partitioned into pairwise disjoint sets $A_{1}, A_{2}, A_{3}$ such that for every $i \in\{1,2,3\},\left|A_{i}\right|=n_{i}$ with $\sum_{a \in A_{i}} a=\nu$ for some element $\nu \in \Gamma$ by Theorem 1.2 or 3.2. Label the vertices from a vertex set $V_{i}$ using elements from the set $A_{i}$ for $i \in\{1,2,3\}$.

Theorem 4.5. Let $G=K_{n_{1}, n_{2}, n_{3}}$ be a complete tripartite graph such that $1 \leq n_{1} \leq n_{2} \leq$ $n_{3}$ and $n_{1}+n_{2}+n_{3}=2^{p}$, then

1. $G$ is $\Gamma$-distance magic for any Abelian group $\Gamma ¥\left(\mathbb{Z}_{2}\right)^{p}$ of order $n$ if and only if $n_{2}>1$,

2. $G$ is $\left(\mathbb{Z}_{2}\right)^{p}$-distance magic if and only if $n_{1} \neq 2$ and $n_{2}>2$.

Proof. Let $G$ have the partition vertex sets $V_{i}$ such that $\left|V_{i}\right|=n_{i}$ for $i \in\{1,2,3\}$.

We can assume that $n_{2}>1$ by Lemma 4.3. If $\left(n_{1}=2\right.$ or $\left.n_{2} \geq 2\right)$ and $\Gamma \cong\left(\mathbb{Z}_{2}\right)^{p}$ then $\Gamma$ does not have a partition $A=\left\{A_{1}, A_{2}, A_{3}\right\}$ such that $\sum_{a \in A_{i}} a=\nu$ for $i \in\{1,2,3\}$ by Theorem 3.2. Thus one can check that then there does not exist a $\Gamma$-distance labeling of $G$.

If $\Gamma \neq\left(\mathbb{Z}_{2}\right)^{p}$ and $n_{i} \geq 2$ for $i \in\{2,3\}$, or $\Gamma \cong\left(\mathbb{Z}_{2}\right)^{p}$ for some integer $p$ and $n_{1} \neq 2$, $n_{2}>2$, then the group $\Gamma$ can be partitioned into pairwise disjoint sets $A_{1}, A_{2}, A_{3}$ such that for every $i \in\{1,2,3\},\left|A_{i}\right|=n_{i}$ with $\sum_{a \in A_{i}} a=\nu$ for some element $\nu \in \Gamma$ by Theorem 3.2, or Lemma 3.1, resp. Label the vertices from a vertex set $V_{i}$ using elements from the set $A_{i}$ for $i \in\{1,2,3\}$.

At the end of this section we put some observations that are implications of Theorem 1.2 for complete $t$-partite graphs. But first we need the following theorem proved in [2] (see Theorem 2.2, [2]).

Theorem 4.6 ([2]). Let $G$ be a graph for which there exists a distance magic labeling $\ell: V(G) \rightarrow\{1,2, \ldots,|V(G)|\}$ such that for every $w \in V(G)$ the following holds: if $u \in N(w)$ with $\ell(u)=i$, then there exists $v \in N(w), v \neq u$, with $\ell(v)=|V(G)|+1-i$. The graph $G$ is a group distance magic graph.

Observation 4.7. Let $G=K_{n_{1}, n_{2}, \ldots, n_{t}}$ be a complete $t$-partite graph such that $1 \leq n_{1} \leq$ $n_{2} \leq \ldots \leq n_{t}$ and $n=n_{1}+n_{2}+\ldots+n_{t}$. Let $\Gamma$ be an Abelian group of order $n$ with exactly three involutions. The graph $G$ is $\Gamma$-distance magic graph if and only if $n_{2}>1$.

Proof. Let $G$ have the partition vertex sets $V_{i}=\left\{x_{1}^{i}, x_{2}^{i}, \ldots, x_{n_{i}}^{i}\right\}$ for $i \in\{1,2, \ldots, t\}$. By Lemma 4.3 we can assume that $n_{2}>1$.

Suppose first that $n_{1}=n_{2}=\ldots=n_{t}=2$. Note that a labeling $\ell: V(G) \rightarrow\{1,2, \ldots, 2 t\}$ defined as $\ell\left(x_{1}^{i}\right)=i, \ell\left(x_{2}^{i}\right)=2 t+1-i$ for $i \in\{1,2, \ldots, t\}$ is distance magic, hence $G$ is 
a group distance magic graph by Theorem 4.6. This implies that there exists a $\Gamma$-distance magic labeling of $G$.

We can assume now that $n_{t} \geq 3$. If $n_{1}>1$, then $n_{t} \geq 4$ or $n_{t-1}=n_{t}=3$. Therefore there exists a zero-sum partition $A_{1}^{\prime}, A_{2}^{\prime}, \ldots, A_{t}^{\prime}$ of the set $\Gamma-\left\{g_{0}\right\}$ such that $\left|A_{t}^{\prime}\right|=n_{t}-1$ and $\left|A_{i}^{\prime}\right|=n_{i}$ for every $1 \leq i \leq t-1$ by Theorem 1.2. Set $A_{t}=A_{t}^{\prime} \cup\left\{g_{0}\right\}$ and $A_{i}=A_{i}^{\prime}$ for every $1 \leq i \leq t-1$. If $n_{1}=1$ then there exists a zero-sum partition $A_{2}^{\prime}, A_{3}^{\prime}, \ldots, A_{t}^{\prime}$ of the set $\Gamma-\left\{g_{0}\right\}$ such that $\left|A_{i}^{\prime}\right|=n_{i}$ for every $2 \leq i \leq t$ by Theorem 1.2. In this case put $A_{1}=\left\{g_{0}\right\}$ and $A_{i}=A_{i}^{\prime}$ for every $2 \leq i \leq t$. Label now the vertices from a vertex set $V_{i}$ using elements from the set $A_{i}$ for $i \in\{1,2, \ldots, t\}$.

Observation 4.8. Let $G=K_{n_{1}, n_{2}, \ldots, n_{t}}$ be a complete $t$-partite graph such that $1 \leq n_{1} \leq$ $n_{2} \leq \ldots \leq n_{t}$ and $n=n_{1}+n_{2}+\ldots+n_{t}$ is odd. The graph $G$ is a group distance magic graph if and only if $n_{2}>1$.

Proof. Let $G$ have the partition vertex sets $V_{i}$ such that $\left|V_{i}\right|=n_{i}$ for $i \in\{1,2, \ldots, t\}$. We can assume that $n_{2}>1$ by Lemma 4.3. If $n_{1}>1$, then $n_{t} \geq 3$. Therefore there exists a zero-sum partition $A_{1}^{\prime}, A_{2}^{\prime}, \ldots, A_{t}^{\prime}$ of the set $\Gamma-\left\{g_{0}\right\}$ such that $\left|A_{t}^{\prime}\right|=n_{t}-1$ and $\left|A_{i}^{\prime}\right|=n_{i}$ for every $1 \leq i \leq t-1$ by Theorem 1.2. Set $A_{t}=A_{t}^{\prime} \cup\left\{g_{0}\right\}$ and $A_{i}=A_{i}^{\prime}$ for every $1 \leq i \leq t-1$. If $n_{1}=1$ then there exists a zero-sum partition $A_{2}^{\prime}, A_{3}^{\prime}, \ldots, A_{t}^{\prime}$ of the set $\Gamma-\left\{g_{0}\right\}$ such that $\left|A_{i}^{\prime}\right|=n_{i}$ for every $2 \leq i \leq t$ by Theorem 1.2. In this case put $A_{1}=\left\{g_{0}\right\}$ and $A_{i}=A_{i}^{\prime}$ for every $2 \leq i \leq t$. Label now the vertices from a vertex set $V_{i}$ using elements from the set $A_{i}$ for $i \in\{1,2, \ldots, t\}$.

Acknowledgments. I thank the anonymous reviewers for their careful reading of our manuscript and their many insightful comments and suggestions.

\section{References}

[1] M. Anholcer, S. Cichacz, Group sum chromatic number of graphs, European J. Combin $\mathbf{5 5}$ (2016) 73-81, doi: 10.1016/j.ejc.2016.02.002.

[2] M. Anholcer, S. Cichacz, I. Peterin, A. Tepeh, Group distance magic labeling of direct product of graphs, Ars Math. Contemp. 9 (2015), 93-108.

[3] S. Cichacz, Note on group distance magic complete bipartite graphs, Cent. Eur. J. Math. 12 (3) (2014) 529-533, DOI: 10.2478/s11533-013-0356-z.

[4] S. Cichacz, Note on group distance magic graphs $G\left[C_{4}\right]$, Graphs Combin. 30 (3) (2014) 565 571, DOI: 10.1007/s00373-013-1294-z.

[5] S. Cichacz, D. Froncek, Distance magic circulant graphs, Discrete Math. 339 (1) (2016) 84-94, DOI: 10.1016/j.disc.2015.07.002.

[6] D. Combe, A.M. Nelson, W.D. Palmer, Magic labellings of graphs over finite abelian groups, Australas. J. Combin. 29 (2004) 259-271.

[7] D. Froncek, Group distance magic labeling of of Cartesian products of cycles, Australas. J. Combin. 55 (2013) 167-174.

[8] G. Kaplan, A. Lev, Y. Roditty, On zero-sum partitions and anti-magic trees, Discrete Math., 2009, 309 (8), 2010-2014, DOI: 10.1016/j.disc.2008.04.012.

[9] M. Miller, C. Rodger and R. Simanjuntak, Distance magic labelings of graphs, Australas. J. Combin., 28 (2003), 305-315.

[10] X. Zeng, On zero-sum partitions of abelian groups. Integers, 15 (2015), Paper No. A44, 16 pp. 